\title{
Tuning the Kinetic Stability of the Amorphous Phase of the Chloramphenicol Antibiotic
}

Sofia Valenti, ${ }^{1,2}$ Michela Romanini, ${ }^{1,2}$ Lourdes Franco,, ${ }^{2,3}$ Jordi Puiggalí, ${ }^{2,3}$ Josep Lluis Tamarit, $^{1,2}$ Roberto Macovez ${ }^{1,2, *}$

${ }^{1}$ Grup de Caracterització de Materials, Departament de Física, Universitat Politècnica de Catalunya, EEBE, Av. Eduard Maristany 10-14, E-08019 Barcelona, Spain.

${ }^{2}$ Barcelona Research Center in Multiscale Science and Engineering, Universitat Politècnica de Catalunya, Campus Diagonal-Besòs, Av. Eduard Maristany 10-14, E-08019 Barcelona, Spain.

3 Synthetic Polymers: Structure and Properties. Biodegradable Polymers, Departament de Enginyeria Química, Universitat Politècnica de Catalunya, EEBE, Av. Eduard Maristany 10-14, E-08019 Barcelona, Spain.

KEYWORDS. Supercooled drug, molecular mobility, crystallization kinetics, chemical degradation, dielectric spectroscopy, polylactic acid. 


\section{ABSTRACT}

We employ broadband dielectric spectroscopy to study the relaxation dynamics and crystallization kinetics of a broad-spectrum antibiotic, chloramphenicol, in its supercooled liquid form. Two dynamic processes are observed: the structural $\alpha$ relaxation, which becomes kinetically frozen at $T_{\mathrm{g}}=302 \pm 1 \mathrm{~K}$, and an intramolecular secondary relaxation. Under isothermal conditions, the supercooled drug displays interconversion between different isomers, followed by recrystallization. Recrystallization follows the Avrami law with Avrami exponent $n$ $=1.3 \pm 0.1$, consistent with a one dimensional growth of crystalline platelets, as observed by electron microscopy. Exposure to humid atmosphere and subsequent heating to high temperature is found to degrade the compound. The partially degraded sample displays a much lower tendency to crystallize, likely because the presence of the degradation products results in spatial frustration. This sample exhibits enhanced conductivity and an additional relaxation, intermediate to the ones observed in the pure sample, which likely corresponds to the noncooperative dynamics of the main degradation product. We find that dispersing the antibiotic in polylactic acid results in an amorphous sample which does not crystallize at room temperature for relatively long times. 
Introduction

Chloramphenicol (hereafter CAP, inset of Figure 1) is a broad-spectrum antibiotic used as a treatment for a number of bacterial infections. Although it had previously fallen into disuse due to limitations on the amount that can be applied not to cause systemic toxicity, it has become an alternative to treat multi-drug-resistant bacteria and has been recently suggested as a potential anticancer drug. ${ }^{1,2}$ CAP blocks protein growth, inhibiting peptide bond formation by binding to a peptidyl transferase enzyme of the bacterial ribosome, which prevents the transfer of aminoacids to the growing peptide chain. Thanks to this general mechanism, it proved effective against Gram-positive as well as Gram-negative (e.g. rickettsiae) bacteria. ${ }^{3}$

CAP is hydrophobic, and shows a low aqueous solubility which limits its absorption rate. If a drug cannot be dissolved in biological fluids fast enough, it does not reach the absorption site and thus it does not trigger the desired biological response. Unfortunately, most drugs are poorly water-soluble, so that additional strategies must be employed to enhance their bioavailability. ${ }^{4}$ Of the various possible approaches to enhance the solubility of active pharmaceutical ingredients, amorphization offers the advantage that it does not require chemical modification of the drug. Solid amorphous (glassy) pure drugs have a proven higher solubility in vivo as well as better dissolution profiles compared with the crystalline form of the drug. , $6,7,8,9,10,11,12^{\text {This is }}$ because the dissolution process is governed by the difference in Gibbs free energy of the initial and final state, i.e., between the separated components and the dissolved state, and the glassy state has higher free energy than the crystalline state, so that dissolution requires less energy, resulting in better rates of uptake by the organism. Several drugs with poor aqueous solubility have reached the drug development stage formulated as amorphous phase. ${ }^{13,14}$ 
On the other hand, glasses are thermodynamically unstable due precisely to their excess in free energy, entropy, and enthalpy, and are therefore susceptible to convert into the more stable and less soluble crystalline phase (by "recrystallization"). A large number of factors (such as preparation method, thermal and mechanical treatments employed during formulation, storage temperature, application of pressure or exposure to humidity, and even the choice of container material) may influence the kinetic stability of the glassy phase, ${ }^{15,16,17}$ and the microscopic mechanism governing metastability remains unclear. The two main aspects governing recrystallization are thermodynamics and molecular kinetics. ${ }^{18}$ The latter is known to influence the crystallization rate: ${ }^{19,20}$ for example, storage well below the glass transition temperature $T_{\mathrm{g}}$ (in fact, below the vitrification temperature of whole-molecule secondary relaxations) prevents recrystallization ${ }^{21,22}$ because, despite the fact that at such relatively low temperature the freeenergy difference between the crystalline and glassy phases (thermodynamic drive) is very large, the molecular mobility is too low for nucleation and growth of the crystalline phase to take place.

In this context, it is important to identify, among drugs with low water solubility such as CAP, those which have sufficiently low recrystallization rate at room temperature so that they can be stored in glassy form for a convenient period of time. It is also interesting to devise strategies to improve the physical stability of those which instead tend to recrystallize from their amorphous state over short times. In the case of CAP, a recent study by means of broadband dielectric spectroscopy ${ }^{23,24}$ has shown that the amorphous phase of CAP can be heated to and maintained at temperatures well above its glass transition temperature $\left(T_{\mathrm{g}}=300 \mathrm{~K}\right)$ without any visible sign of recrystallization. ${ }^{25}$ This result is very interesting in view of the low aqueous solubility of this antibiotic, and it is moreover puzzling in light of the fact that an earlier study ${ }^{26}$ on CAP has instead shown that recrystallization above $T_{\mathrm{g}}$ proceeds so fast that complete crystallization is 
observed in calorimetry thermograms obtained while heating from the glass state at a rate of 10 $\mathrm{K} / \mathrm{min}$ already at $T_{\mathrm{g}}+25 \mathrm{~K}$.

Recrystallization of a supercooled liquid can be hindered through spatial frustration, since in such case the molecules cannot aggregate to form an ordered structure. For example, twocomponent amorphous mixtures have been shown to remain stable even at temperatures where both pure components recrystallize (the first studies of binary mixtures go back to the 1960's ${ }^{27,28}$ ). The authors of the afore-mentioned recent study of kinetically stable supercooled liquid samples of CAP have proposed that the metastability of their samples may be due to spatial frustration associated with the presence, in the sample, of different tautomeric forms of the drug. ${ }^{25}$ We show here that the observed metastability of CAP is indeed due to spatial frustration, but that it is due to the presence of hydrolysis products. We find evidence for the same tautomeric transformations also in supercooled liquid samples of CAP that do crystallize.

We find that recrystallization of pure CAP takes place on the timescale of hours at temperatures higher than $T_{\mathrm{g}}$. At room temperature (just below $T_{\mathrm{g}}$ ) glassy CAP is found to crystallize in a timespan of days, which indicates that refrigeration would be needed to prevent the amorphous drug to crystallize. The crystallization kinetics follows the Avrami law with exponent $n$ equal to 1.3, corresponding to one-dimensional (fibrillar) growth with only sporadic nucleation. On the other hand, we show that the metastability of amorphous CAP can indeed be tuned employing different strategies. We find that humid CAP degrades when heated above its melting temperature (degradation of CAP was previously observed when the drug was exposed to chlorine and UV radiation or in aqueous media ${ }^{29,30,31,32,33}$ ). The resulting glass, which is in fact a mixture of CAP and degradation products, does not seem to crystallize over short times. These findings can rationalize the recent report of a lack of recrystallization of supercooled 
liquid CAP. ${ }^{25}$ If it can be shown that the hydrolysis products are unharmful, then this strategy may be a simple and viable one to obtain kinetically stable amorphous CAP samples. We show moreover that CAP remains amorphous also when mixed with a biocompatible polymer such as polylactic acid, likely due to spatial frustration.

\section{Materials and Methods}

Chloramphenicol $\left(\mathrm{C}_{11} \mathrm{H}_{12} \mathrm{Cl}_{2} \mathrm{~N}_{2} \mathrm{O}_{5}, \mathrm{M}_{\mathrm{w}}=323.13 \mathrm{~g} / \mathrm{mol}\right)$ was purchased from Sigma-Aldrich with a purity higher than $98 \%$ and stored in air at ambient temperature. Three types of CAP samples were measured. In one case the CAP powder was initially dried in a vacuum oven for 24 $\mathrm{h}$ at room temperature and then melted and rapidly cooled to obtain a glass. In the second case, the powder was melted directly after retrieval from its storage bottle in air. In order to check the effect of water on crystallization, a third type of CAP sample was prepared by melting an ovendried sample and then allowing it to crystallize in water-vapor saturated atmosphere. For broadband dielectric spectroscopy (BDS) experiments, the powder was melted directly between the stainless steel electrodes of the capacitor (details are given in the following paragraph) and cooled at a rate of approximately $10 \mathrm{~K} / \mathrm{min}$. For differential scanning calorimetry (DSC) experiments, the powder was either melted directly during the first DSC scan (first and second type), or melted outside in the DSC pan (third type). Finally, binary mixtures of CAP with polylactic acid (PLA) were obtained by dissolving PLA (grade 4032D, from Natureworks LLC, USA, a semi-crystalline material with a concentration of D enantiomers less than $2 \%$ ), in chloroform and letting it dissolve overnight using a magnetic stirrer at $310 \mathrm{~K}$. The correct amount of CAP to achieve a drug loading of $20 \mathrm{wt}-\%$ in PLA was then dissolved in acetone and such solution was then mixed with the PLA/chloroform mixture. Films were prepared by solvent casting directly on metal disk electrodes, followed by heating to $433 \mathrm{~K}$ to allowall solvents to 
evaporate and to melt the crystalline fraction of PLA formed during solvent evaporation. Characterization by X-ray diffraction showed the obtained mixture to be amorphous, and to remain so during long periods of time of storage at room temperature.

Differential scanning calorimetry (DSC) experiments were carried out on different CAP samples in open aluminum pans (diameter $4 \mathrm{~mm}$ ) under nitrogen atmosphere, by means of a Q100 calorimeter from TA Instruments. Measurements were performed using a heating/cooling rate of $10 \mathrm{~K} / \mathrm{min}$ and drug mass around $10 \mathrm{mg}$.

Infrared absorption spectra were recorded in the $4000-600 \mathrm{~cm}^{-1}$ range with a Fourier Transform FTIR 4100 Jasco spectrometer equipped with a Specac model MKII Golden Gate attenuated total reflection (ATR) cell.

A Novocontrol Alpha analyzer was used for broadband dielectric spectroscopy (BDS) measurements. The sample was placed in a stainless steel parallel-plate capacitor (diameter of $1.2 \mathrm{~cm}$ ) specially designed for the analysis of liquid samples, with the two electrodes kept at fixed distance by means of silica spacers of $50 \mu \mathrm{m}$ diameter. Temperature control of the capacitor and thus of the sample was achieved with a nitrogen-gas flow cryostat with an error not higher than $0.3 \mathrm{~K}$. Two different types of BDS experiments were carried out, in order to study the relaxation dynamics and the crystallization kinetics of the material. In the first case, the previously dried or as-stored polycrystalline powder sample was placed in the capacitor, melted at $423 \mathrm{~K}$ and quenched to cryogenic temperature inside the cryostat. Successive isothermal measurements were done every $10 \mathrm{~K}$, from 133.2 to $233.2 \mathrm{~K}$, every $5 \mathrm{~K}$, from 243.2 to $298.2 \mathrm{~K}$, every $2 \mathrm{~K}$, from 303.2 to $328.2 \mathrm{~K}$, and again every $5 \mathrm{~K}$, from 333.2 to $373.2 \mathrm{~K}$. All measurements were done in the frequency $(v)$ range between $10^{-2}$ and $10^{6} \mathrm{~Hz}$. 
The second type of BDS experiment, designed to study the isothermal crystallization kinetics of CAP, consisted of measuring the decrease of strength of the dielectric loss as a function of time during the crystallization of the supercooled material at different (fixed) temperatures. To this end, the molten sample was placed inside a precooled cryostat at $293.2 \mathrm{~K}$ (i.e., about $8 \mathrm{~K}$ below $T_{\mathrm{g}}$ ) and then the temperature was raised at a rate of $10 \mathrm{~K} / \mathrm{min}$ until reaching the desired isothermal crystallization temperature. We measured the recrystallization at five distinct temperatures between 319 and $335 \mathrm{~K}$ by acquiring several dielectric spectra one after another, in the frequency range from 1 to $10^{6} \mathrm{~Hz}$. A freshly prepared sample was used for each isothermal recrystallization experiment.

To obtain relaxation time values and quantify the changes in relaxation dynamics, we fitted the dielectric spectra (real and imaginary part simultaneously) with a power law for the conductivity contribution and a superposition of Havriliak-Negami functions ${ }^{34,35}$ for the relaxation peaks. The analytical expression of the Havriliak-Negami function is:

(eq. 1) $\varepsilon^{*}(\omega)=\varepsilon_{\infty}+\frac{\Delta \varepsilon}{\left(1+\left(i \omega \tau_{H N}\right)^{a}\right)^{b}}$

Here $\omega=2 \pi \nu$ is the angular frequency, $\varepsilon_{\infty}$ is the permittivity in the high frequency limit, $\Delta \varepsilon$ is the dielectric strength, the exponents $a$ and $b$ (which may vary between 0 and 1 ) describe the symmetric and asymmetric broadening of the peak, respectively, and $\tau_{\mathrm{HN}}$ is a time parameter connected to the characteristic relaxation time $\tau_{\max }$, corresponding to the maximum of the relaxation time distribution. $\tau_{\max }$ was calculated from the fitting parameters as:

(eq. 2) $\tau_{\max }=\tau_{H N}\left[\sin \left(\frac{a \pi}{2 b+2}\right)\right]^{-1 / a}\left[\sin \left(\frac{a b \pi}{2 b+2}\right)\right]^{1 / a}$ 
Specific cases of this relaxation are the Cole-Cole (CC) ${ }^{36}$ and Cole-Davidson (CD) ${ }^{37}$ relaxations, which are obtained for $b=1$ and $a=1$, respectively. For both cases $\tau_{\mathrm{max}}=\tau_{\mathrm{HN}}$. Most secondary peaks are broad but symmetric, and are often described with the Cole-Cole function.

\section{Results and Discussion}

The blue line in Figure 1a represents the DSC thermogram of the pure (vacuum-dried) CAP powder, measured upon heating. The observed onset of melting is $422 \pm 1 \mathrm{~K}$, in agreement with previous findings, ${ }^{26}$ with a enthalpy of fusion of $103 \pm 3 \mathrm{~J} / \mathrm{g}$. X-ray diffraction patterns of the vacuum-dried powder are in agreement with previous findings. ${ }^{38}$ The glass transition temperature, identified in the second heat-up scan acquired after cooling the melted sample (Figure 1b), is $300 \pm 2 \mathrm{~K}$. No recrystallization peak is detected, in contrast with an earlier calorimetry study. $^{26}$ 


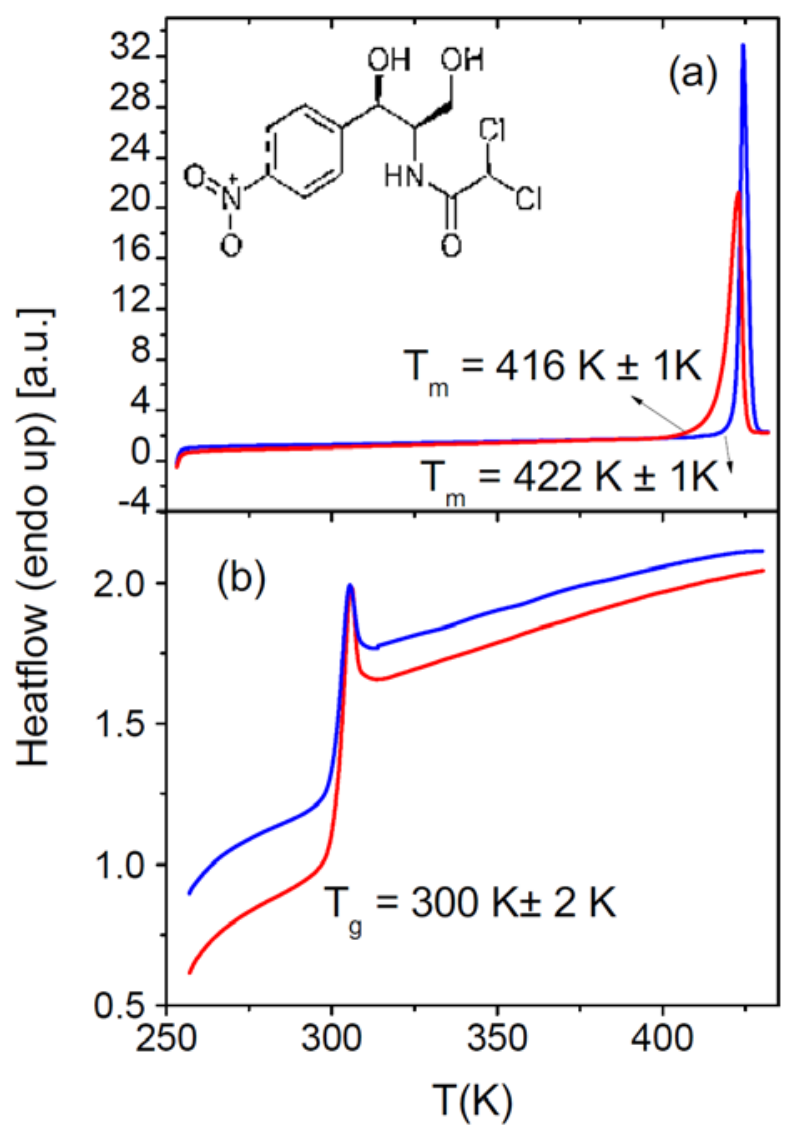

Figure 1. DSC thermograms, obtained by heating at $10 \mathrm{~K} / \mathrm{min}$, of pure CAP (blue) and CAP recrystallized from the glass in humid atmosphere and stored under humid conditions for 3 days (red). The upper panel shows the melting run, where the onset of melting is indicated. The lower panel shows the second run after melting, where the glass transition temperature is indicated. Inset to upper panel: molecular structure of CAP.

A second sample was prepared by melting the powder in an open DSC pan, cooling the resulting liquid to the glassy state and then storing it for 3 days in a water-vapor saturated atmosphere. The resulting sample had fully recrystallized (red line in Figure 1a). The melting temperature of the sample recrystallized in the presence of water saturated atmosphere is $416 \pm 1$ $\mathrm{K}$ and the enthalpy of fusion is only $87 \pm 3 \mathrm{~J} / \mathrm{g}$. The X-Ray diffraction patterns of this recrystallized phase are virtually identical to those of the pure vacuum-dried powder, showing 
that no hydrate phase ${ }^{39}$ was formed in the sample recrystallized in water saturated atmosphere. The lower melting point and enthalpy observed in Figure 1a are likely due to a decrease of the purity of the sample. Full recrystallization was observed also in glassy samples stored in air at room temperature over a period of few days. These results confirm that cold recrystallization of CAP is possible even in the glassy state, that is, that glassy CAP is unstable against the formation of the crystal phase.

We employed dielectric spectroscopy to study the molecular relaxation dynamics of amorphous CAP (both in the supercooled liquid and glassy state). The vacuum-dried CAP powder was melted and fast-cooled to $133 \mathrm{~K}$, and isothermal spectra were then acquired by stepwise increasing the temperature. Figure 2 shows the dielectric loss spectra at selected temperatures. Panel b shows the spectra above room temperature, in which the structural relaxation is visible on top of a conductivity background which increases towards low frequencies. This relaxation is the-so called primary $\alpha$ process, which corresponds to the cooperative motion of CAP molecules and is associated with the glass transition. The peak maximum of the $\alpha$ relaxation moves to lower frequencies the lower the temperature, indicating decreasing molecular mobility with decreasing thermal energy, and it is below $0.01 \mathrm{~Hz}$ for $T<$

$302 \mathrm{~K}$, in agreement with the calorimetric $T_{\mathrm{g}}{ }^{26}{ }^{2}$ Figure 2a shows the spectra below $T_{\mathrm{g}}$, where a secondary $(\gamma)$ relaxation much less intense than the $\alpha$ process is observed.

The primary relaxation was fitted to a Havriliak-Negami function, and the best fit was obtained with a Cole-Davidson function ( $a=1$ ), with $b$ varying monotonically between 0.59 and 0.65 . The secondary relaxation was fitted with a Cole-Cole function $(b=1)$ and the resulting shape parameter $a$ varied between 0.18 and 0.3 . 


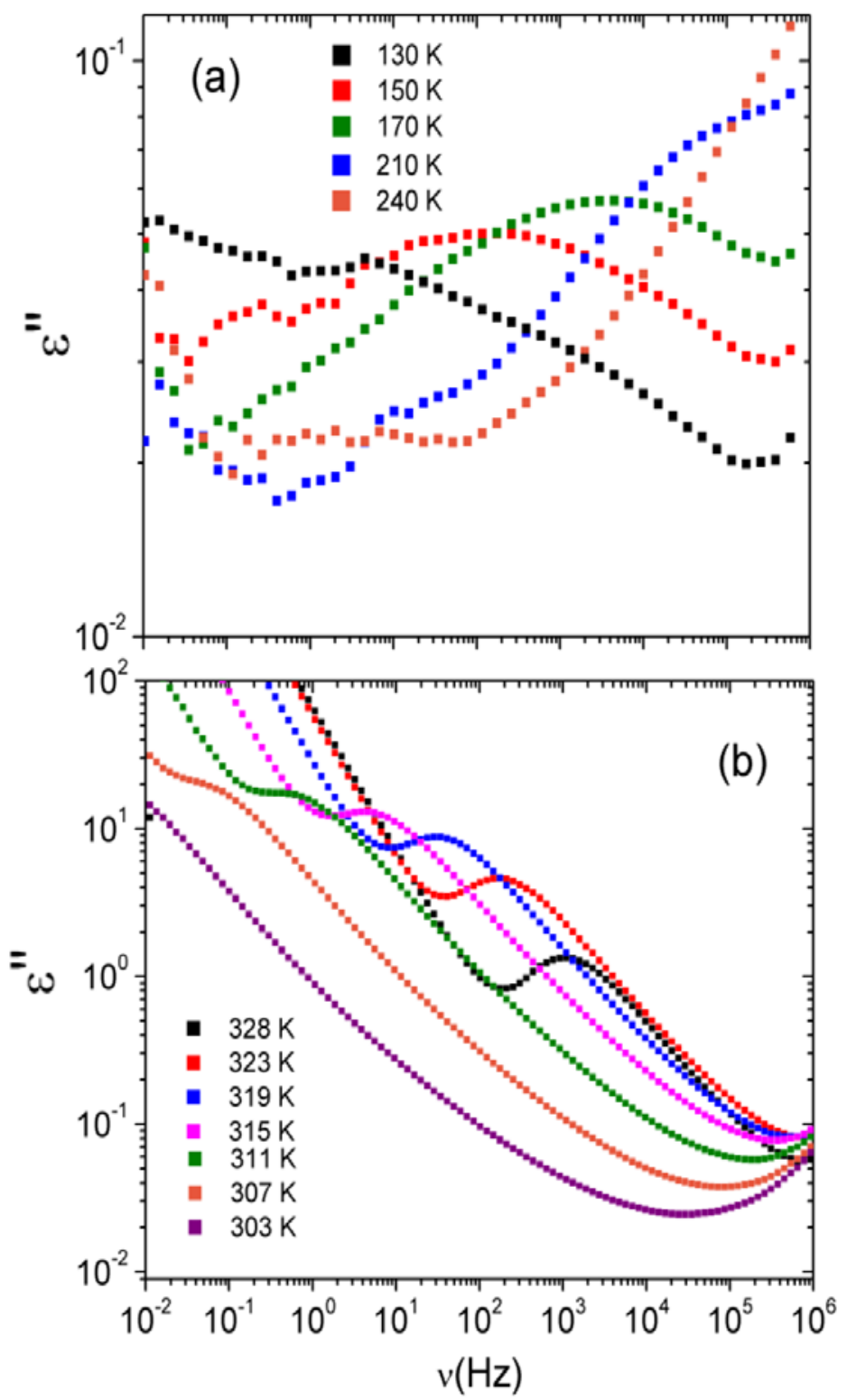

Figure 2. Selected dielectric loss spectra of pure CAP between 133 and 243 K (a) and between 303 and $328 \mathrm{~K}$ (b). Low frequency data in (a) have been smoothed.

The relaxation times of both processes are displayed as Arrhenius plot in Figure 3. The inset to Figure 3 displays the relaxation strength of the $\alpha$ relaxation for temperatures just above $T_{\mathrm{g}}$. It can be seen that the relaxation strength of the $\alpha$ process scales as $1 / T$, as expected from the Kirkwood-Fröhlich equation. ${ }^{34}$ The secondary relaxation is a simply-activated process, following 
the Arrhenius law with activation energy $E_{a}=38.0 \pm 0.6 \mathrm{~kJ} / \mathrm{mol}$, a relatively low value which is close to that reported in ref 25 and which suggests an intramolecular origin of this relaxation process, ${ }^{40}$ as also discussed below. Instead, the temperature dependence of the $\alpha$ relaxation time could be modeled with a Vogel-Fulcher-Tamman (VFT) function, defined by:

(eq. 3) $\log \tau_{\alpha}=\log \tau_{\infty}+\frac{1}{\ln 10} \frac{D T_{0}}{T-T_{0}}$

Here $\tau_{\infty}$ is the high temperature limit of the relaxation time, $D$ is the so-called strength parameter (proportional to the inverse of the fragility index, see below) and $T_{0}$ is the VogelFulcher temperature, whose value indicates the departure from a simply-activated behavior. The obtained values were $\log \left(\tau_{\infty} /[\mathrm{s}]\right)=-15.0 \pm 0.7, D=7.6 \pm 0.8, T_{0}=253 \pm 3 \mathrm{~K}$. The estimated glass transition temperature, defined as customary as the temperature at which $\tau_{\alpha}$ reaches $100 \mathrm{~s}$, was $302.0 \pm 0.5 \mathrm{~K}$, in agreement with the DSC results of Figure 1 .

From the fit we can obtain the fragility index of amorphous CAP, which is defined as

(eq. 4) $m=\left.\frac{d\left(\log \tau_{\alpha}\right)}{d\left(T_{g} / T\right)}\right|_{T=T_{g}}$

The fragility value obtained from the slope of a linear fit near $T_{\mathrm{g}}$ is $m=104 \pm 2$, which is in agreement with the one calculated directly from the fit parameters $m=(D / \ln 10) \cdot\left(\left(T_{0} / T_{\mathrm{g}}\right) /(1-\right.$ $\left.\left.T_{0} / T_{\mathrm{g}}\right)^{2}\right)=(1.1 \pm 0.2) \cdot 10^{2}$. CAP is hence a rather fragile glass-former.

The corresponding $\alpha$ relaxation times and VFT parameters reported in ref. 25 are slightly different; in particular, the authors of this work found a fragility of $m=116$. This discrepancy is probably related to the fact that the sample characterized by Paluch and coworkers was partially degraded, as discussed in the following. 


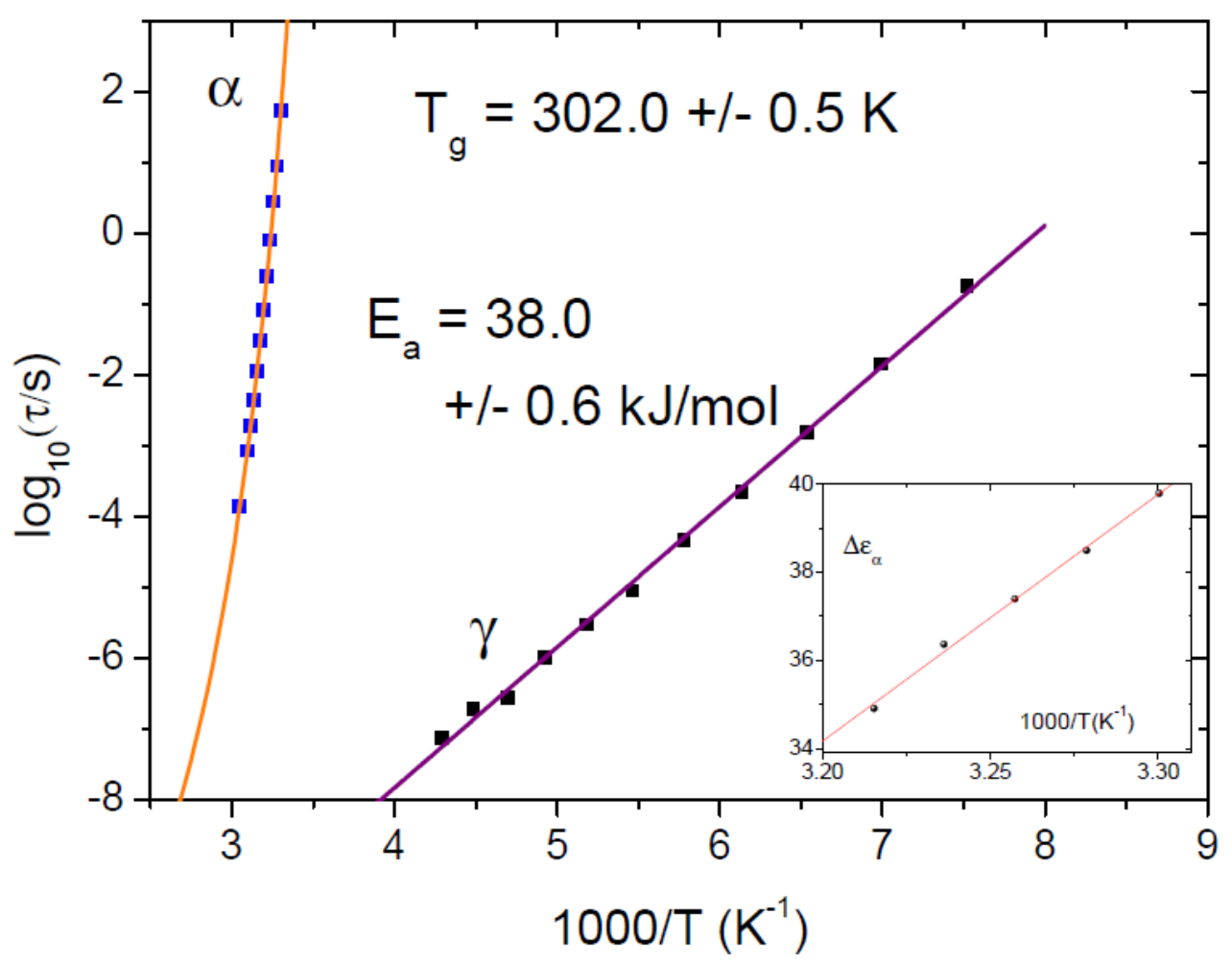

Figure 3. Relaxation map of pure CAP. Blue (resp., black) squares represent the $\alpha$ (resp., $\gamma$ ) relaxation. Continuous lines are fits with a VFT function (orange line) and an Arrhenius function (purple line), respectively. Inset: Temperature dependence of the dielectric strength of the $\alpha$ relaxation below the onset temperature of recrystallization.

The relaxation dynamics could not be explored at higher temperature. At $315 \mathrm{~K}$, in fact, a clear decrease of the loss peak maximum is visible (Figure 2b), indicative of the occurrence of recrystallization. The intensity of the relaxation (dielectric strength) is directly proportional to the number of relaxing dipoles in the sample ${ }^{34}$ and recrystallization implies loss of orientational motions. Since the viscosity is lower at higher temperatures, recrystallization, whose kinetics is mostly governed by molecular diffusion, is more likely to take place above $T_{\mathrm{g}}$. This rationalizes the fact that the decrease in dielectric strength is only observed at high temperatures, where crystallite nucleation and growth take place and molecular dipoles become immobilized. ${ }^{41,42}$ 


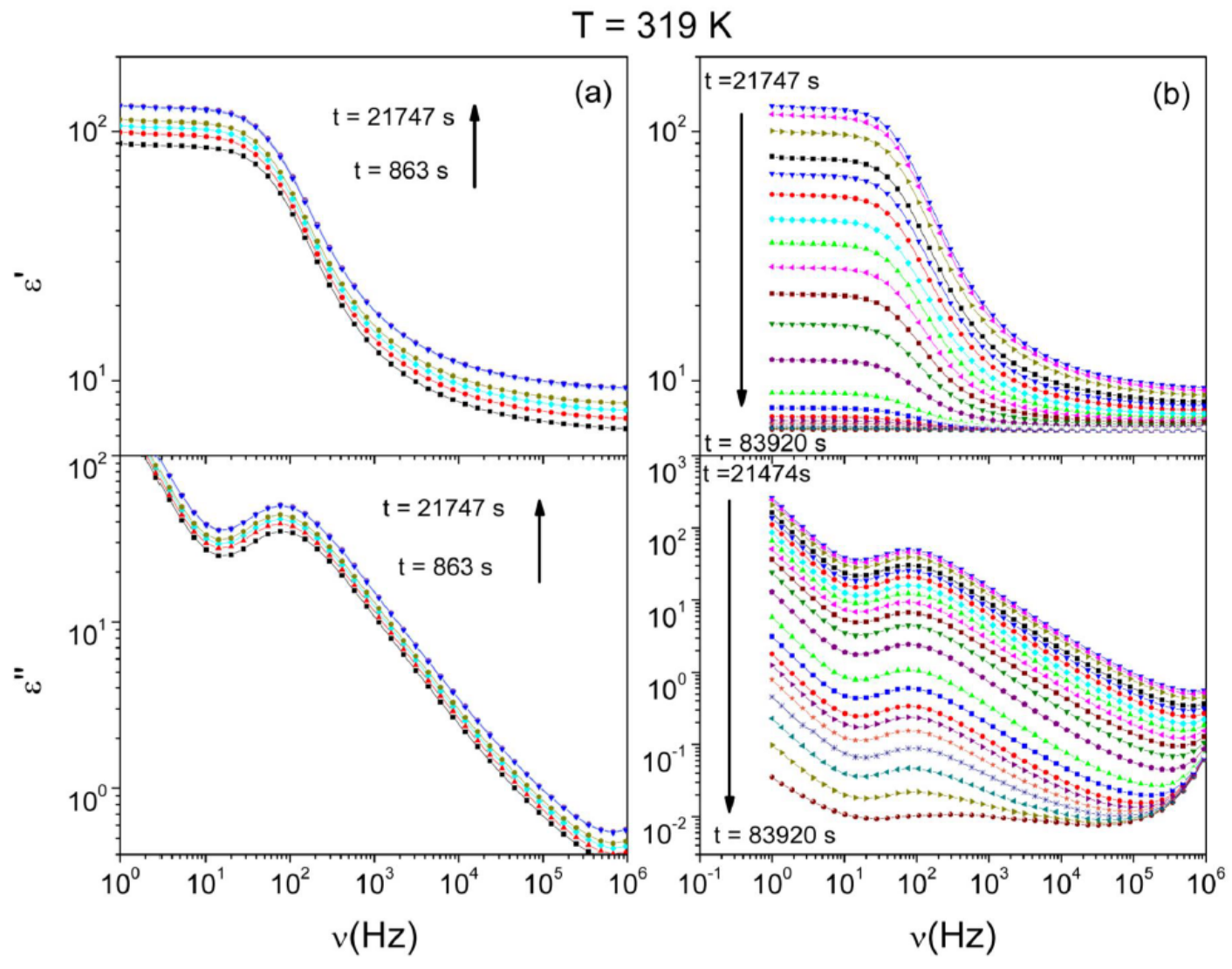

Figure 4. Dielectric spectra of the real (top panels) and imaginary part (bottom panels) of the dielectric permittivity of CAP at $319 \mathrm{~K}$ at different times as indicated, during isothermal tautomerization (a) and subsequent recrystallization (b).

We performed isothermal recrystallization measurements to investigate the characteristic timescale and mechanism of crystal growth from the supercooled liquid phase. Figure 4 show dielectric spectra as a function of time, recorded at $319 \mathrm{~K}$. Similar to what was reported in ref 25 , we observe an initial increase in the spectral intensity (in the first isothermal spectra recorded, see Figure 4a), which is most likely due to the tautomerization process proposed in ref 25, which involves transformation between three distinct molecular isomers of CAP. The dielectric strength is proportional to the number of dipolar molecules in the sample times the square of the dipole moment; since the total number of molecules in the sample is not changing, the only possible 
explanation of the increase in the strength is that the molecular dipole changes, as already argued in ref 25. The origin of the increase of dielectric strength is then ascribed to an increase in the molecular dipole moment as the population of tautomers changes over time. In particular, by the authors of ref 25 suggested two paths of tautomerization: the amide-imidic acid and the keto-enol tautomerization. According to their DFT study, of the three tautomers, the enol form has the highest molecular dipole, the amide/keto form has the lowest dipole moment, while the imidic acid form has a value of dipole moment that is intermediate between the former two. It would thus appear that the number of enol and/or imidic acid tautomers is increasing with time as liquid CAP is supercooled and held at isothermal conditions above $T_{\mathrm{g}}$. The origin of the tautomerization process is likely the different equilibrium value of the tautomer concentrations above the melting point (concentrations which are likely maintained during the rapid quench to below $T_{\mathrm{g}}$ ) and in the supercooled liquid state below the melting point. ${ }^{25}$

After tautomer transformations have ended, i.e., after a stationary static permittivity value is reached, one can observe, in the same isothermal series of spectra, the decrease of spectral intensity with time due to recrystallization, a decrease visible both in the real (top panel of Figure 4b) and in the imaginary (bottom panel) part of the dielectric spectra. This observation rules out the hypothesis, brought forth in ref 25 , that that the changes induced in the sample by isomeric transformation of CAP are sufficient to hinder its recrystallization.

The evolution of crystallization is best analyzed looking at the dielectric strength $\Delta \varepsilon$, which as mentioned is directly correlated to the number of relaxing molecules. Because the real and imaginary parts of the permittivity are related by Kramers-Kronig transformation, the dielectric strength of a relaxation (area below the peak in the loss spectrum) is $\Delta \varepsilon=\varepsilon_{\mathrm{s}}-\varepsilon_{\infty}$, that is, it is 
equal to the difference between the static (real) permittivity $\varepsilon_{\mathrm{s}}$ and the high frequency limit of the real part of the permittivity, $\varepsilon_{\infty}$. The so-obtained dielectric strength is shown as function of time in Figure 5a. The width of the relaxation was found to grow over time (as the $b$ parameter of the Cole-Davidson function lowers, not displayed) probably indicating that, while recrystallization develops, the scattering in the distribution of sizes of the amorphous domains increases. ${ }^{43}$

The crystallization kinetics can be followed through the normalized static permittivity difference, defined as: ${ }^{44}$

(eq. 5) $\varepsilon_{n}(t)=\frac{\varepsilon_{S}(L)-\varepsilon_{S}(t)}{\varepsilon_{S}(L)-\varepsilon_{S}(C)}$

where $\varepsilon_{\mathrm{s}}(\mathrm{L})$ is the static permittivity of the (supercooled) liquid and $\varepsilon_{\mathrm{s}}(\mathrm{C})$ is the static permittivity of the crystal, while $\varepsilon_{\mathrm{s}}(t)$ is the static dielectric permittivity of the sample measured at the time $t$ from the start of the isothermal measurement. For each isothermal recrystallization temperature, the values of $\varepsilon_{\mathrm{s}}$ were taken at a distinct fixed frequency in the range between 1 and $10 \mathrm{~Hz}$, but always corresponding to the low frequency plateau value of $\varepsilon$ '. 

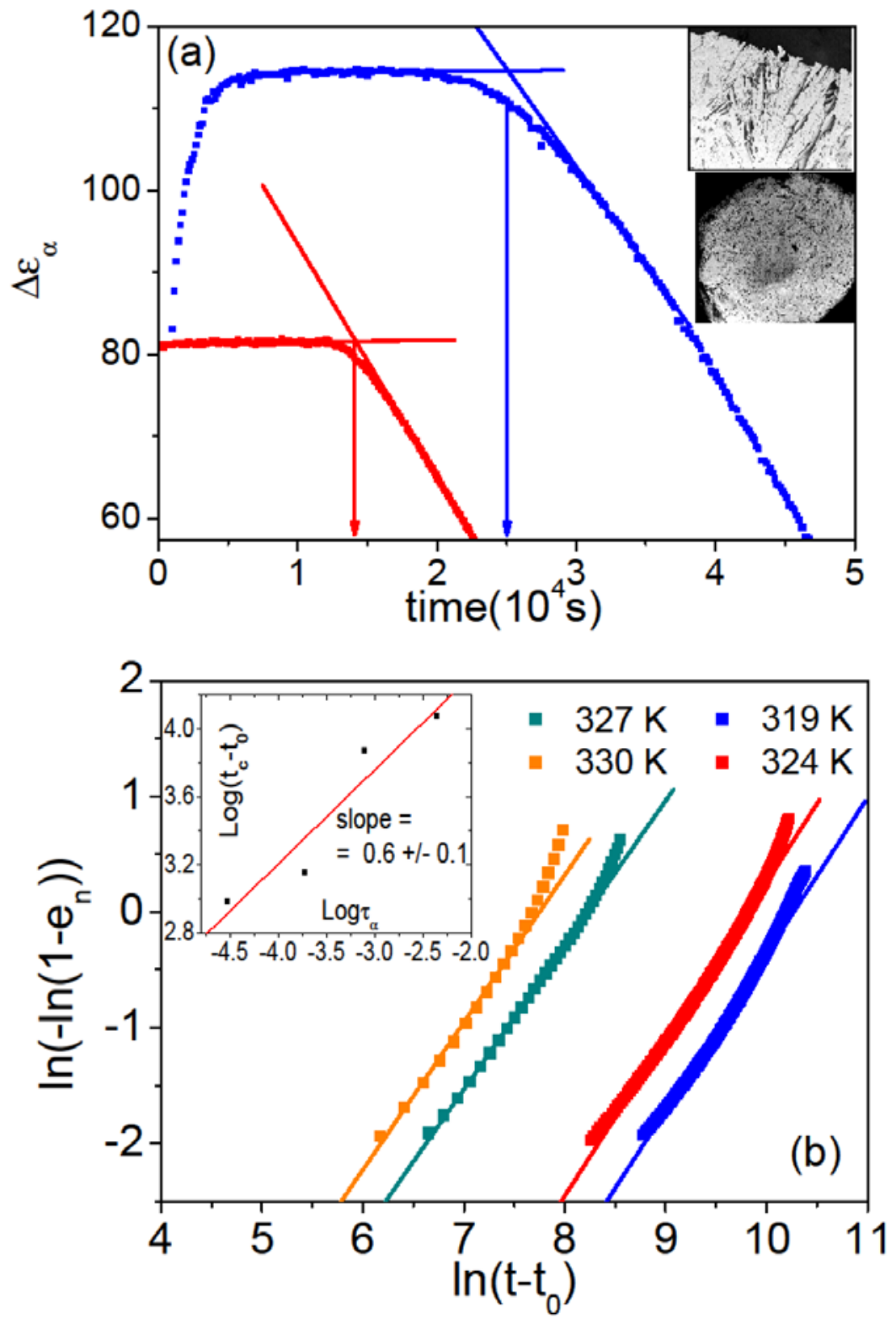

Figure 5. (a) Dielectric strength $\Delta \varepsilon_{\alpha}$ of the structural relaxation of CAP as a function of time for the isotherms at 319 (blue) and $324 \mathrm{~K}$ (red).The arrows indicate the onset time of crystallization $\left(t_{0}\right)$. Inset: scanning electron microphotographs of CAP crystallites formed by recrystallization from the melt. (b) Avrami plot of isothermal experiments at 319 (blue), 324 (red), 327 (cyan), and $330 \mathrm{~K}$ (orange). Lines represent linear fits. Inset: correlation of the crystallization time $t_{\mathrm{c}}-t_{\mathrm{o}}$ with the structural relaxation time $\tau_{\alpha}$. 
Assuming a temporal dependence of recrystallization as described by Avrami, ${ }^{45,46} \varepsilon_{\mathrm{n}}$ is given by: ${ }^{47}$

(eq 6) $\varepsilon_{n}(t)=1-\exp \left(-K\left(t-t_{\mathrm{o}}\right)^{n}\right)$

Here, $t_{\mathrm{o}}$ is the onset (induction) time of nucleation, $K$ is the rate of recrystallization and $n$ is the Avrami exponent which depends only on the morphology of crystallites and the mechanism of the recrystallization process. ${ }^{48}$ The value of $t_{0}$ is estimated taking the intercept of the horizontal plateau of $\Delta \varepsilon$ at short times with the tangent to the decreasing portion of the graph of $\Delta \varepsilon$ (see Figure 5a). Figure 5b shows the plot of $\ln \left(-\ln \left(1-\varepsilon_{n}\right)\right)$ versus $\ln \left(t-t_{0}\right)$ for four isothermal measurements. The observed linear dependence confirms the validity of Avrami’s law. The slope of the fit gives the parameter $n$, and the intercept is $\ln (K)$. The Avrami exponent is $n=1.3 \pm 0.1$, and it is constant (within experimental error) for all four isothermal conditions considered. A value in this range indicates predominant growth of the crystal nuclei in one dimension, in the form of dentritic structures of needle-like to rod-like or even laminar crystallites. This is confirmed by the insets to Figure 5a, which displays two SEM microphotographs of CAP crystallites formed by recrystallization of the supercooled liquid. Similar crystallite shapes were reported also in previous studies on the as-synthesized CAP powder. ${ }^{49}$

The inset of Figure $5 \mathrm{~b}$ shows the relation between $\log \tau_{\alpha}$ and $\log \left(t_{\mathrm{c}}-t_{0}\right)$, where $t_{\mathrm{c}}$ is the characteristic crystal growth time, defined as the time at which $\varepsilon_{\mathrm{n}}$ becomes equal to 0.25 . Though not precise, the linear regression gives a slope value of $0.6 \pm 0.1$. This correlation between the structural relaxation time and the time of crystallization has been observed before (albeit with a slightly different slope) in several systems, for example in the case of stiripentol ${ }^{20}$ and of indomethacine and celecoxib, ${ }^{50}$ and also in binary mixtures of pharmaceutical compounds and 
polymeric excipients. ${ }^{51}$ The correlation between crystal growth rate and mobility (or equivalently, viscosity) above $T_{\mathrm{g}}$ appears to be a general feature of glass formers (also nonpharmaceutical ones), ${ }^{41},{ }^{52}, 53$ related to the fact that the crystallization rate is kinetically driven near the glass transition temperature.

As mentioned, previous dielectric measurements ${ }^{25}$ at ambient pressure showed no recrystallization of CAP, which was ascribed to the effect of conformational changes (tautomerization) in the supercooled liquid sample. The authors of this previous study report that no crystallization takes place at $323 \mathrm{~K}$ during at least two and a half days, and they report that no crystallization takes place at $338 \mathrm{~K}$ during at least three hours. Instead, Figure 5 shows that pure CAP crystallizes relatively fast at $324 \mathrm{~K}$, and our experiments at $335 \mathrm{~K}$ (not shown) indicate that crystallization at this temperature is so fast that it starts well before reaching the isothermal temperature conditions. As we show in the following, the most likely explanation of the failure of CAP to recrystallize in the experiments reported in ref 25 is its partial degradation.

Previous studies have shown that CAP has two main degradation pathways, namely, amide hydrolysis and carbon-chlorine cleavage. ${ }^{29,30,31,32}$ In the former case the reaction is acid-base catalyzed (though its rate does not depend on the $\mathrm{pH}$ of the concentration) while in the latter case, $\mathrm{pH}$ and temperature play a significant role. In the former case, the products of degradation are 1-(p-nitrophenyl)-2-amino-propanediol and dichloroacetic acid, while in the latter case they are a Cl-substituted alcohol CAP derivative and (at least partially) dissociated hidrochloric acid, ${ }^{30}$ but other secondary pathways that lead to degradation may exist. ${ }^{29}$ Furthermore it was reported that, while water-free preparations show no sign of degradation (pure CAP does not degrade upon melting ${ }^{54}$ ), detectable amounts of these degradation products are present in aqueous solutions as well as in preparations of CAP with relatively low amounts of water (e.g., 
condensed from humid air). Recent studies ${ }^{5,56}$ report that the half-life of CAP in water at $333 \mathrm{~K}$ is of the order of 20 days, and that in boiling water CAP exhibits $12 \%$ degradation in 1 hour.

Dielectric measurements on the as-stored CAP powder (without prior drying in the vacuum oven) indicate that it indeed does not recrystallize, or else recrystallizes at a much slower rate, than the pure (vacuum dried) material. Figure 6a shows isothermal dielectric measurements at 324 Kon a supercooled liquid sample of CAP melted at $433 \mathrm{~K}$ directly after retrieval from the storage bottle (in air). After $768 \mathrm{~min}, \Delta \varepsilon$ as detected from the real permittivity spectra is lowered by only $5 \%$ with respect to its value at the beginning of the experiment, while no clear difference is detected in the imaginary permittivity (loss) spectra. After $977 \mathrm{~min}$ a slight difference is observed in the loss spectra, and $\Delta \varepsilon$ is lowered only by $8 \%$. This is clearly in contrast to what is observed in Figures 4 and 5: for example, when the vacuum-dried sample is melted at $423 \mathrm{~K}$, isothermal crystallization at $324 \mathrm{~K}$ starts already after an induction time $t_{0}=230 \mathrm{~min}$. 

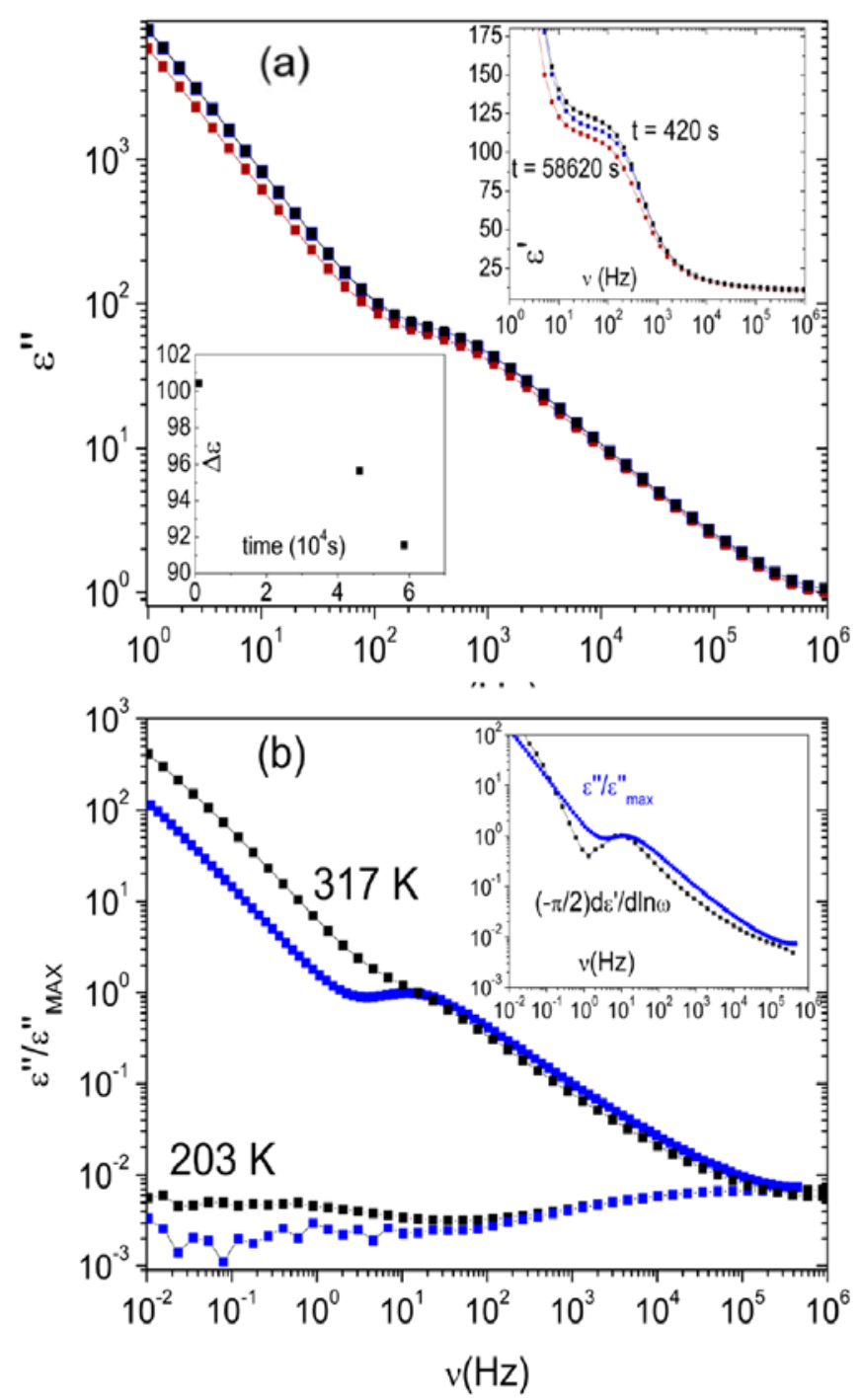

Figure 6. (a) Dielectric loss spectra of CAP measured at $324 \mathrm{~K}$, respectively 7 (black), 768 (blue) and 977 (red) minutes after reaching this temperature from the glassy state. The upper inset shows the corresponding $\varepsilon$ ' spectra, while lower inset shows the dielectric strength as function of time. (b) Comparison between the dielectric loss of the partially degraded (black) and pure CAP (blue), at two temperatures, above (317 K) and well below (203 K) the glass transition temperature. Inset: comparison at $317 \mathrm{~K}$ between the dielectric loss spectrum of the pure sample and the Kramers-Kronig transformation of the real permittivity spectrum of the degraded sample. 
A possible rationale for the hindering of recrystallization could be that heating the initially water containing powder leads to partial degradation, and that the products of degradation impede the ordering of the CAP molecules into a crystalline phase. In other words, just like many binary mixtures fail to recrystallize due to the extremely slow formation kinetics (or lack) of a co-crystal phase, it can be expected that CAP does not recrystallize when mixed with the products of degradation such as for example the 1-(p-nitrophenyl)-2-amino-propanediol molecules. The occurrence of partial degradation was confirmed by direct observation of the sample at the end of the experiment: while pure CAP is white, the outcome of melting the asstored CAP sample was a viscous yellow-brownish liquid, with a color similar to that previously reported for sunlight degraded CAP. ${ }^{29}$ To analyze the outcome of degradation, we have recorded the FTIR spectrum of the degraded sample (after annealing for $3600 \mathrm{~s}$ at $433 \mathrm{~K}$ ) and compared it to the spectrum of the pure (vacuum-dried) sample. As can be seen from Figure 7, the amide A (3130-3500 $\mathrm{cm}^{-1}$ ) and amide II (around $1500 \mathrm{~cm}^{-1}$ ) bands were modified after annealing, with the degraded sample showing much broader and less defined peaks. The first amide band shows clearly how hydrogen bonding network is affected by the high temperature treatment, as the two defined peaks that are visible in the pure sample at 3258 and $3343 \mathrm{~cm}^{-1}$ merge into a broader and wider band.

We therefore suggest that the observed stability of supercooled CAP obtained by melting the as-stored powder is indeed due to spatial frustration, but not due to the presence of different tautomers, as suggested in ref 25, and instead due to the presence of hydrolysis products. Tautomerization transformations are observed also in pure (non-degraded) CAP, and they result in samples that crystallize above $T_{\mathrm{g}}$ over relatively short periods of time. Hence it would appear that the presence of different tautomers is not an impediment for nucleation of the crystal phase. 


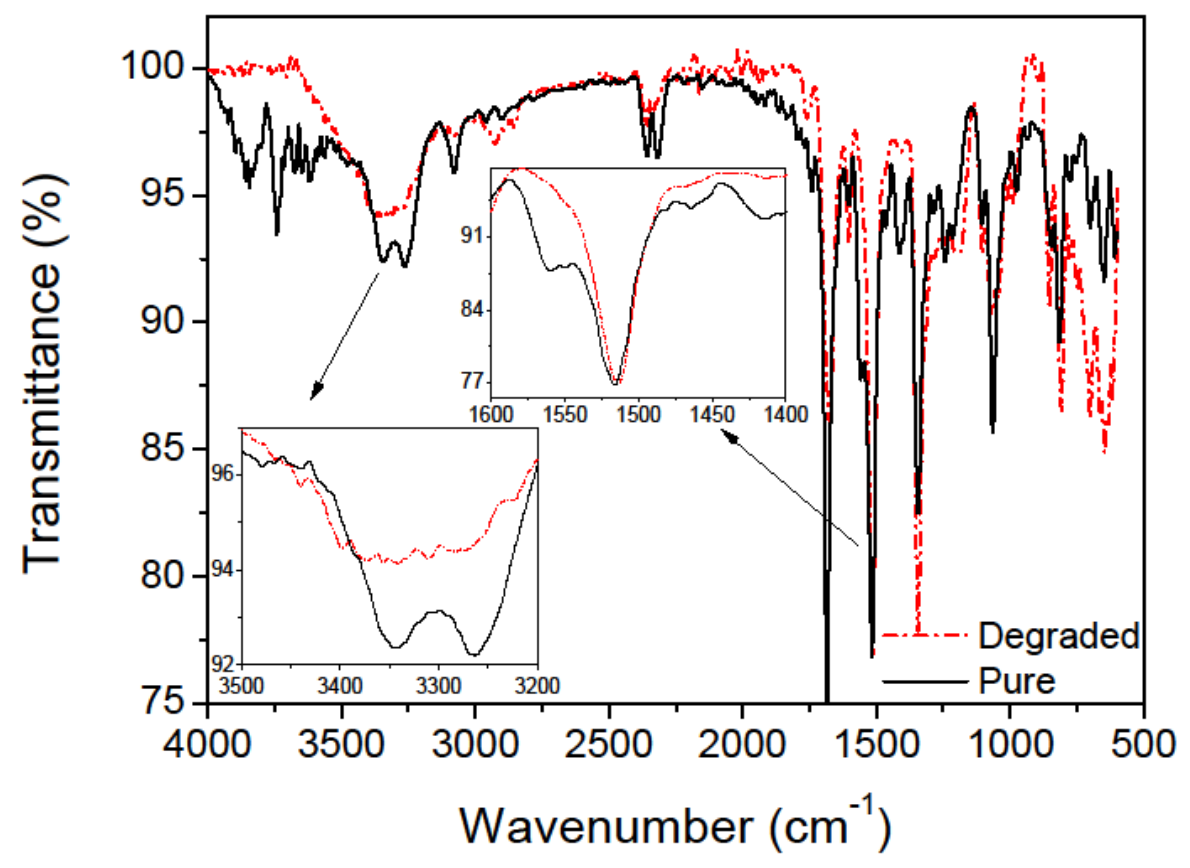

Figure 7. Comparison of the FTIR spectra of pure CAP (black) and degraded CAP (red dotted line).

To assess how degradation influences the dynamics and allow further comparison with the results of ref 25, we performed dielectric spectroscopy measurements on a (partially) degraded sample. This sample displayed the same primary and secondary relaxation as pure CAP (Figure 8a). As can be seen from Figure 6b, the loss spectrum of the degraded sample was characterized by a higher conductivity, most likely due to charged products of the water-induced partial decomposition (such as protons and hydroxyl and chlorine anions), which lowers the visibility of the structural relaxation peak. In order to allow a better comparison of the spectra, the inset of Figure $6 \mathrm{~b}$ displays the approximate Kramers-Kronig transformation of the real part of the dielectric constant, ${ }^{57,58}$ together with the loss spectrum of the non-degraded sample at the same temperature. The relaxation times are virtually identical. This can be clearly observed in the 
comparison of the relaxation maps of both samples, displayed in Figure 8a. Both the structural relaxation times and those of the secondary relaxation in the glassy state are seen to coincide roughly in both samples (and with the relaxation times reported in ref 25, not shown), indicating that the quantity of degraded molecule is not enough to affect the cooperative dynamics of the sample.

On the other hand, the partially degraded sample also exhibits another secondary relaxation, which is absent in non-degraded CAP, as visible directly in the comparison of the respective spectra at $203 \mathrm{~K}$ shown in the inset to Figure 8a. The activation energy of this (slower) secondary process is $\mathrm{E}_{\mathrm{a}}=65.0 \pm 0.8 \mathrm{~kJ} / \mathrm{mol}$. In the main panel we show the prediction of the so-called Coupling Model, which has been found to describe in many instances the properties of the Johari-Goldstein relaxation of molecular glass formers. ${ }^{59,60}$ The relaxation time of the secondary relaxations is far from that predicted by the Coupling Model, which shows that neither is a Johari-Goldstein relaxation. Hence the additional dynamic process observed in the partially degraded sample must have a different origin.

The observed activation energy and relaxation times suggest that this process could be due to the (non-cooperative) relaxation dynamics of degradation products present as impurities in the sample. The 1-(p-nitro-phenyl)-2-amino-propanediol moieties, for example, are present in lower concentration and have lower mass than CAP, which would rationalize why the new relaxation follows an Arrhenius behavior with faster times compared with the structural ones. Similarly, as mentioned previously, the presence of ionic degradation products rationalizes the increase in conductivity observed in partially degraded CAP. Figure 8b shows the Arrhenius plot of the dc conductivity $\left(\sigma_{\mathrm{dc}}\right)$ of both pure and partially degraded CAP, identified as the plateau value of the real part of the conductivity spectra (defined by the relation $\sigma^{\prime}(\omega)=\omega \varepsilon_{0} \varepsilon^{\prime \prime}(\omega)$ ). 

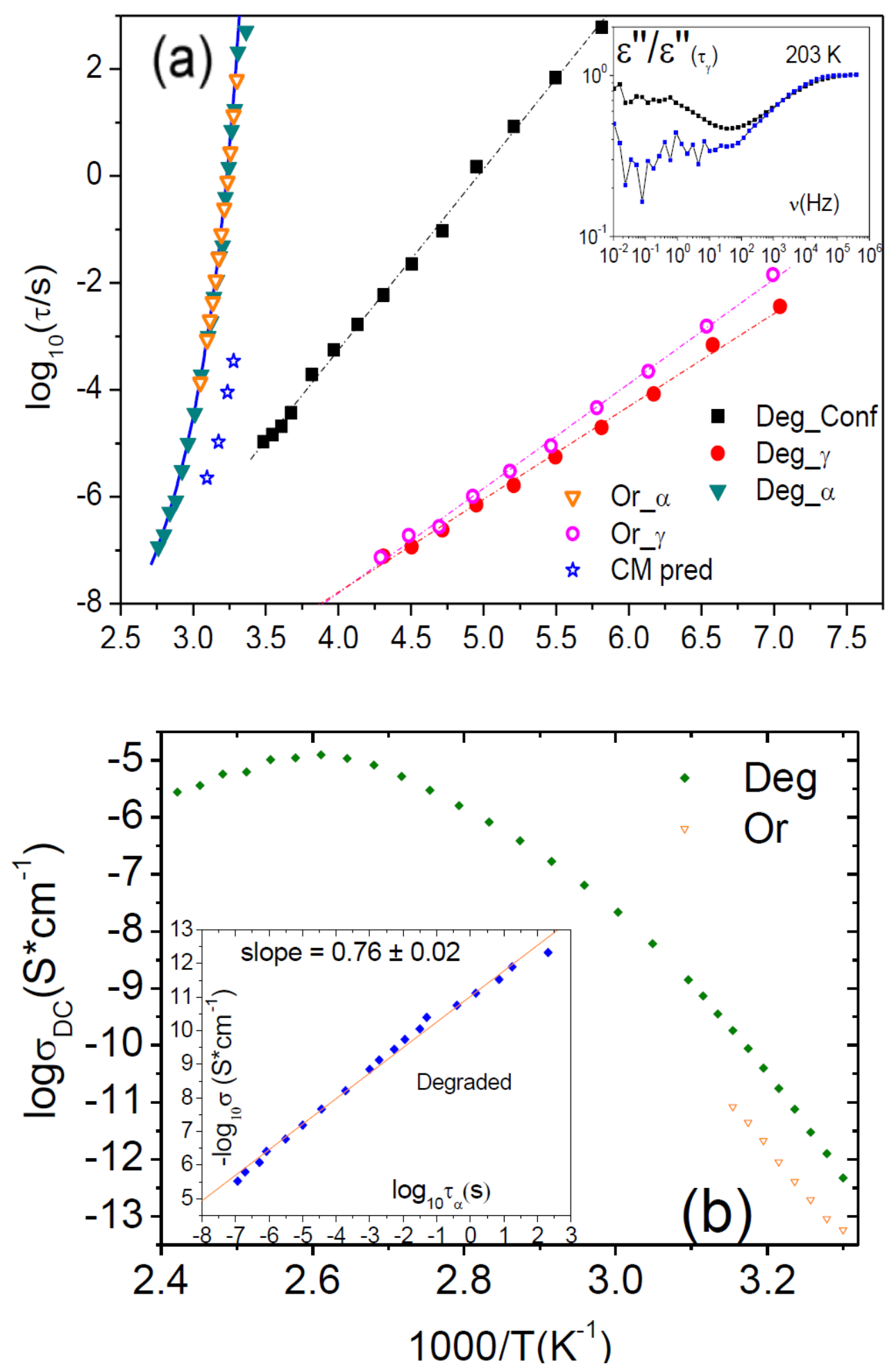

Figure 8. (a) Relaxation map of the pure (open symbols) and partially degraded (filled symbols) amorphous CAP samples. Triangles represent the $\alpha$ relaxation, while squares and circles 
secondary relaxations. The solid line is the fit with the VFT eq. 3, while dashed lines represent Arrhenius fits of secondary relaxation times. Open stars represent the relaxation time of the Johari-Goldstein relaxation predicted by the Coupling Model. Inset: comparison of the loss spectra of the pure and degraded sample at $T=203 \mathrm{~K}$. (b) Arrhenius plot of the dc conductivity of both amorphous samples. Inset: sublinear dependence between the dc conductivity and the relaxation time of the structural process in the partially degraded sample.

The dc conductivity of the partially degraded sample is significantly higher, it does not exhibit a monotonic behavior (in fact, it decreases above $384 \mathrm{~K}$ ), and, as visible in the inset to Figure 7b, it does not scale linearly with the cooperative relaxation time (rather, it displays a power law correlation with it, referred to fractional Stokes-Einstein rule ${ }^{61}$ ). These last two observations suggest that the conductivity enhancement is due to relatively small, ionic species (for example, chlorine ions, hydroxyl ions, or protons) whose diffusion is decoupled from the structural relaxation of the much bulkier CAP molecules, ${ }^{62}$ and which likely recombine into neutral species upon heating to above approximately $380 \mathrm{~K}$. Other glass-forming systems show a decoupling of ion conduction and structural relaxation, in particular ionic liquids, where various processes are responsible for conduction. ${ }^{63}$

Even though the partially degraded pharmaceutical is stable in the amorphous phase, another approach should be taken to stabilize the amorphous form of CAP, so as to take advantage of its better dissolution profile with respect to the crystalline form, and at the same time avoid the potential toxicity of the degradation products. A strategy that suggests itself in the case of CAP is to mix it with another (biocompatible) substance that could induce spatial frustration as in the partially degraded sample. ${ }^{64}$ To demonstrate the feasibility of this approach, we dispersed CAP (20\% in weight) in a commercial biopolymer, namely poly-lactic acid (PLA), which due to its 
biocompatibility, degradation into natural metabolites and low cost, is one of the most widely used materials in biomedical applications. ${ }^{65,66}$

The left inset to Figure 9 shows the DSC thermogram of the resulting amorphous solid-state dispersion, whose $T_{\mathrm{g}}$ is observed to be $316 \mathrm{~K}$, in between the $T_{\mathrm{g}}$ of pure PLA (332 K) and that of CAP (302 K). At higher temperature, only the recrystallization and subsequent melting of PLA can be seen. Interestingly, both the $T_{\mathrm{g}}$ and melting temperature of PLA seem to be affected by the presence of the drug. The onset of PLA recrystallization occurs at relatively high temperature (ca. $380 \mathrm{~K}$ ), hence it does not affect the storage and metastability of the amorphous pharmaceutical at ambient temperature. As visible in the dielectric spectra displayed in Figure 9, at a given temperature the structural relaxation peak of the mixture is observed at a frequency in between the peak of pure PLA and the peak of pure CAP, as expected from the observed variation in $T_{\mathrm{g}}$. No phase separation is observed: only one $T_{\mathrm{g}}$ is detected in DSC, and only one structural loss peak is observed in BDS. The right inset of Figure 9 shows the Arrhenius plot for the three samples, where again the relaxation times of the dispersion are seen to lie in between the relaxation times of the polymer and of pure CAP. This totally amorphous and homogeneous sample was stable at ambient temperature for at least a few weeks. 


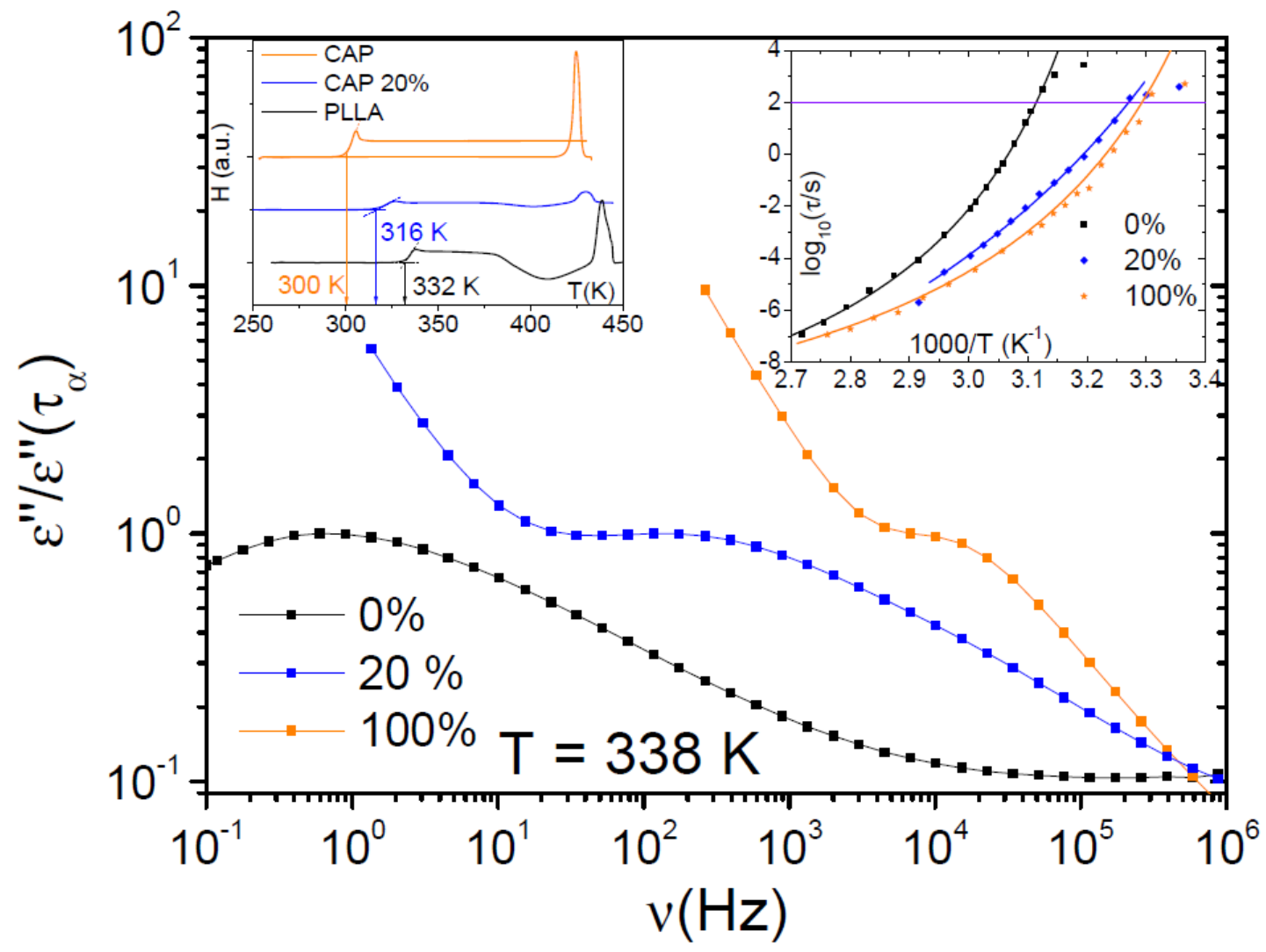

Figure 9. Comparison of the loss spectra at 338 of pure PLA (black), pure CAP (orange) and a $20 \%$ weight dispersion of CAP in PLA (blue), normalized to $\varepsilon$ '” $\left(\tau_{\alpha}\right)$. Lines are guides to the eye. Right inset: Arrhenius plot of the structural relaxation time of the same three samples. Lines are VFT fits of the corresponding relaxation times. Left inset: DSC traces for the 20\% CAP mixture, pure CAP and pure PLA ( $T_{\mathrm{g}}$ is indicated). The baseline was subtracted from the traces.

\section{Conclusions}

We have measured the molecular relaxation dynamics and crystallization kinetics of the Chloramphenicol antibiotic. Dielectric characterization shows a fragile liquid, with dynamic glass transition temperature $T_{\mathrm{g}}=300 \mathrm{~K}$ and $m=104$, characterized by the presence of a pronounced structural relaxation $(\alpha)$ and a weaker secondary relaxation $(\gamma)$. Recrystallization 
kinetics experiments and SEM characterization indicate that the supercooled liquid recrystallizes into dendritic structures, with Avrami exponent of $n=1.3 \pm 0.1$. Prior to crystallization, a tautomer interconversion reaction takes place; this shows that the tautomerism of CAP does not per se prevent recrystallization of the amorphous compound, contrary to the results of ref 25.

If the CAP sample contains initially some water and is heated to above the melting point, partial degradation results, which hinders recrystallization. A non-monotonous temperature dependence of the ionic conductivity is observed in the degraded sample, suggesting the presence of small-ion species. The degraded sample displays, besides the $\alpha$ and $\gamma$ relaxations, also a third intermediate relaxation process, probably associated to the local dielectric relaxation of degradation products in the sample. This sample showed much slower recrystallization kinetics, which suggests that obtaining binary mixtures of CAP constitutes a possible path to avoid degradation while still metastabilizing the amorphous state. Hence the kinetic stability of some supercooled liquid CAP samples does indeed result from spatial frustration as suggested in ref 25, however, the spatial frustration is not a consequence of different tautomers but rather of hydrolysis products. If these can be shown not to be harmful, then melting and cooling CAP powder exposed to air can be employed as a straightforward and facile approach to obtain kinetically stable amorphous CAP. We further explored the effect of spatial frustration by dispersing CAP in poly-lactic acid: the structural relaxation time and $T_{\mathrm{g}}$ of the resulting mixture are intermediate between those of the two pure components, and the dispersion is found to be stable in the amorphous phase for relatively long periods of time. 


\section{AUTHOR INFORMATION}

\section{Corresponding Author}

* Author to whom correspondence should be addressed. Electronic mail: roberto.macovez@upc.edu.

\section{Author Contributions}

The manuscript was written through contributions of all authors. All authors have given approval to the final version of the manuscript.

\section{ACKNOWLEDGMENT}

This work has been partially supported by the Spanish Ministry of Economy and Competitiveness MINECO through project FIS2017-82625-P and through the project MAT2015-69547-R, and by the Generalitat de Catalunya under project 2017SGR-42.

\section{ABBREVIATIONS}

CAP, chloramphenicol; VFT, Vogel-Fulcher-Tamman; PLA, poly-lactic acid. 


\section{TOC GRAPHICS}
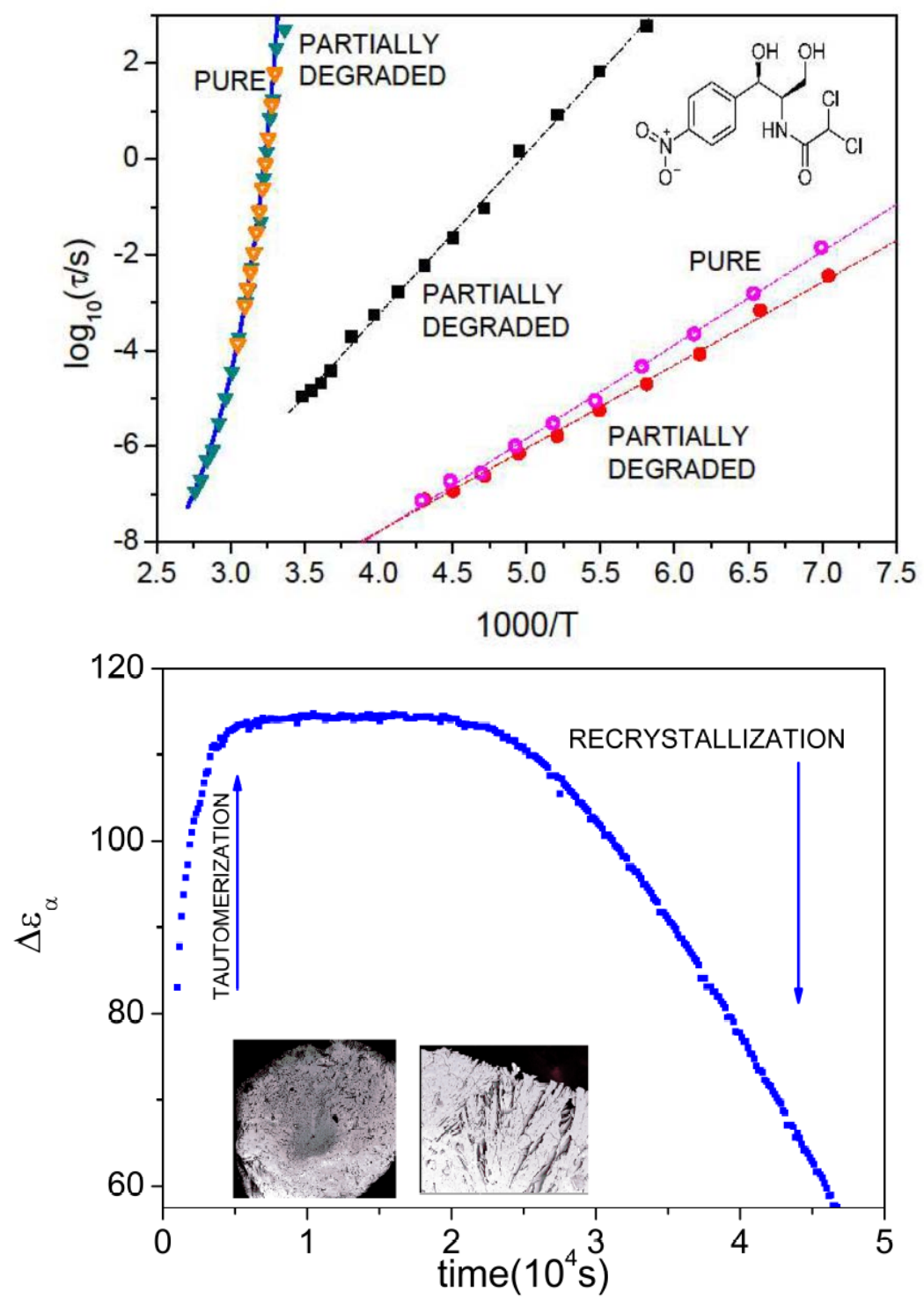


\section{REFERENCES}

${ }^{1}$ Kostopoulou, O. N.; Kouvela, E. C.; Magoulas, G. E.; Garnelis, T.; Panagoulias, I.;Rodi, M.;Kalpaxis, D. L. Conjugation with Polyamines Enhances the Antibacterial and Anticancer Activity of Chloramphenicol. Nucleic Acids Res. 2014, 42, 8621-8634.

2 Tian, F.; Wang, C.; Tang, M., Li, J.; Cheng, X.; Zhang, S.; Li, H. The Antibiotic Chloramphenicol May Be an Effective New Agent for Inhibiting the Growth of Multiple Myeloma. Oncotarget 2016, 7, 51934-51942.

3 Monro, R. E.; Vazquez D. Ribosome-Catalysed Peptidyl Transfer: Effects of Some Inhibitorsof Protein Synthesis. J. Mol. Biol. 1967, 28, 161-165.

4 Williams, H. D.; et al. Strategies to Address Low Drug Solubility in Discovery and Development. Pharmacol. Rev. 2013, 65, 315-499

5 Murdande, S. B.; Pikal, M. J.; Shanker, R. M.; Bogner, R.H. Aqueous Solubility of Crystalline and Amorphous Drugs: Challenges in Measurement. Pharm. Dev. Technol. 2011, 16, 187-200.

6 Gupta, P.; Chawla, G.; Bansal, A. Physical Stability and Solubility Advantage from Amorphous Celecoxib: the Role of Thermodynamic Quantities and Molecular Mobility. Mol. Pharm. 2004, 1, 406-413.

${ }^{7}$ Serajuddin, A. Solid Dispersion of Poorly Water-Soluble Drugs: Early Promises, Subsequent Problems, and Recent Breakthroughs. J. Pharm. Sci. 1999, 88, 1058-1066. 
8 Hancock, B. C.; Parks, M. What Is the True Solubility Advantage for Amorphous Pharmaceuticals? Pharm. Res. 2000, 17, 397-404.

${ }^{9}$ Craig, D.; Royall, P.; Kett, V.; Hoptopn, M. The Relevance of the Amorphous State to Pharmaceutical Dosage Forms: Glassy Drugs and Freeze Dried Systems. Int. J. Pharm. 1999, 179, 179-207.

${ }^{10}$ Babu, N. J.; Nangia, A. Solubility Advantage of Amorphous Drugs and Pharmaceutical Cocrystals. Cryst. Growth. Des. 2011, 11, 2662-2679.

${ }^{11}$ Laitinen, R.; Löbmann, K.; Strachan, C. J.; Grohganz, H.; Rades, T. Emerging Trends in the Stabilization of Amorphous Drugs. Int. J. Pharm. 2013, 453, 65-79.

${ }^{12}$ Kalepu, S.; Nekkanti, V. Insoluble Drug Delivery Strategies: Review of Recent Advances and Business Prospects. Acta Pharm. Sin. B 2015, 5, 442-453.

13 Qian, F.; Tao, J.; Desikan, S.; Hussain, M.; Smith, R. Mechanistic Investigation of Pluronic ${ }^{\circledR}$ Based Nano-Crystalline Drug-Polymer Solid Dispersions. Pharm. Res. 2007, 24, 1551-1560.

${ }^{14}$ Wu, T.; Yu, L. Surface Crystallization of Indomethacin Below Tg. Pharm. Res. 2003, 23, 2350-2355.

${ }^{15}$ Patterson, J.; James, M.; Forster, A.; Lancaster, R.; Butler, J.; Rades, T. The Influence of Thermal and Mechanical Preparative Techniques on the Amorphous State of Four Poorly Soluble Compounds. J. Pharm. Sci. 2005, 94, 1998-2012. 
${ }^{16}$ Yu, L. Amorphous Pharmaceutical Solids: Preparation, Characterization and Stabilization. Adv. Drug Deliv. Rev. 2001, 48, 27-42.

${ }^{17}$ Martínez, L. M.; Videa, M.; López-Silva, G. A.; de los Reyes, C. A.; Cruz-Angeles, J.; González, N. Stabilization of Amorphous Paracetamol Based Systems Using Traditional and Novel Strategies. Int. J. Pharm. 2014, 477, 294-305.

${ }^{18}$ Graeser, K.; Patterson, J.; Zeitler, J.; Gordon, K.; Rades, T. Correlating Thermodynamic and Kinetic Parameters with Amorphous Stability. Eur. J. Pharm. Sci., 2009, 37, 492-498.

19 Grzybowska, K.; Capaccioli, S.; Paluch, M. Recent Developments in the Experimental Investigations of Relaxations in Pharmaceuticals by Dielectric Techniques at Ambient and Elevated Pressure. Adv. Drug Deliv. Rev. 2016, 100, 158-182.

${ }^{20}$ Ruiz, G. N.; Romanini, M.; Barrio, M.; Tamarit, J.L.; Pardo, L.C.; Macovez, R. Relaxation Dynamics vs Crystallization Kinetics in the Amorphous State: the Case of Stiripentol. Mol. Pharm. 2017, 14, 3636-3643.

${ }^{21}$ Capen, R.; Christopher, D.; Forenzo, P.; Ireland, C.; Liu, O.; Lyapustina, S.; O’Neill, J.; Patterson, N.; Quinlan, M.; Sandell, D.; Schwenke, J.; Stroup, W.; Tougas, T. On the Shelf Life of Pharmaceutical Products. AAPS PharmSciTech. 2012, 13, 911-918.

${ }^{22}$ Kissi, E. O.; Grohganz, H.; Löbmann, K.; Ruggiero, M. T.; Zeitler, J. A.; Rades, T. GlassTransition Temperature of the $\beta$-Relaxation as the Major Predictive Parameter for Recrystallization of Neat Amorphous Drugs. J. Phys. Chem. B 2018, 122, 2803-2808. 
${ }^{23}$ Adrjanowicz, K.; Zakowiecki, D.; Kaminski, K.; Hawelek, L.; Grzybowska, K.; Tarnacka, M.; Paluch, M.; Cal, K. Molecular Dynamics in Supercooled Liquid and Glassy States of Antibiotics: Azithromycin, Clarithromycin and Roxithromycin Studied by Dielectric Spectroscopy. Advantages Given by the Amorphous State. Mol. Pharm. 2012, 9, 1748-1763.

${ }^{24}$ Kaminski, K.; Adrjanowicz, K.; Wojnarowska, Z.; Dulski, M.; Wrzalik, R.; Paluch, M.; Kaminska, E.; Kasprzycka, A. Do Intermolecular Interactions Control Crystallization Abilities of Glass-Forming Liquids? J. Phys. Chem. B 2011, 115, 11537-11547.

${ }^{25}$ Knapik-Kowalczuk, J.; Wojnarowska, Z.; Chmiel, K.; Rams-Baron, M.; Tajber, L.; Paluch, M. Can Storage Time Improve the Physical Stability of Amorphous Pharmaceuticals with Tautomerization Ability Exposed to Compression? The Case of Chloramphenicol Drug. Mol. Pharm. 2018, 15, 1928-1940.

${ }^{26}$ Ford, J. L. The Use of Thermal Analysis in the Study of Solid Dispersions. Drug Dev. Ind. Pharm. 1987, 13, 1741-1777.

${ }^{27}$ Smyth, C. P. Microwave Absorption and Molecular Structure in Liquids. XVII. Dielectric Relaxation Times and Shapes of Rigid Molecules. Proc. Natl. Acad. Sci.1956, 42, 234-239.

${ }^{28}$ Hassell, W. F.; Walker, S. Dielectric Studies. Part 11 - Relaxation Processes of Six Monosubstituted Benzenes in Dilute Solution. Trans. Faraday Soc. 1966, 62, 2695-2703.

${ }^{29}$ Shih, I. K. Degradation Products of Chloramphenicol. J. Pharm. Sci. 1971, 60, 786-787. 
${ }^{30}$ Higuchi, T; Bias, C. D. The Kinetics of Degradation of Chloramphenicol in Solution: I. A Study of the Rate of Formation of Chloride Ion in Aqueous Media. J. Am. Pharm. Ass. 1953, 42, 707-714.

${ }^{31}$ Higuchi, T.; Marcus, A. D.; Bias, C. D. The Kinetics of Degradation of Chloramphenicol in Solution: II. Over-All Disappearance Rate from Buffered Solutions. J. Am. Pharm. Ass. 1954, 43, 129-134.

${ }^{32}$ Higuchi, T.; Marcus, A. D. The Kinetics of Degradation of Chloramphenicol in Solution: III. The Nature, Specific Hydrogen Ion Catalysis, and Temperature Dependencies of the Degradative Reactions. J. Am. Pharm. Ass. 1954, 43, 530-535.

33 Dong, H.; Qiang, Z.; Hu, J.; Qu, J. Degradation of Chloramphenicol by UV/Chlorine Treatment: Kinetics, Mechanism and EnhancedFormation of Halonitromethanes. Water Res. 2017, 121, 178-185.

34 Böttcher, C. J. F.; Bordewijk, P. Theory of electric polarization, Vol. 2, Elsevier Science Ltd, 1978.

${ }^{35}$ Havriliak, S.; Negami, S. A Complex Plane Representation of Dielectric and Mechanical Relaxation Processes in Some Polymers. Polymer 1967, 8, 161-210.

${ }^{36}$ Cole, R. H.; Cole, K. S. Dispersion and Absorption in Dielectrics II. Direct Current Characteristics. J. Chem. Phys. 1942, 10, 98-105.

${ }^{37}$ Davidson, D.; Cole, R. Dielectric Relaxation in Glycerol, Propylene Glycol, and n-Propanol. J. Chem. Phys. 1951, 19, 1484-1490. 
${ }^{38}$ Dunitz, J. D. The Crystal Structure of Chloramphenicol and Bromamphenicol. J. Am. Chem. Soc. 1952, 74, 995-999.

39 Zachariah, M.; Mitsari, E.; Romanini, M.; Zygouri, P.; Gournis, D.; Barrio, M.; Tamarit, J. Ll.; Macovez, R. Water-Triggered Conduction Mediated by Proton Exchange in a Hygroscopic Fulleride and its Hydrate. J. Phys. Chem. C 2015, 119, 685-694.

${ }^{40}$ Adrjanowicz, K.; Wojnarowska, Z.; Grzybowska, K.; Hawelek, L.; Kaminski, K.; Paluch, M.; Kasprzycka, A.; Walczak, K. Molecular Dynamics and Crystallization Phenomenon of Supercooled and Glassy DNA and RNA Nucleosides: $\beta$-Adenosine, $\beta$-Thymidine, and $\beta$ Uridine. Phys. Rev. E 2011, 84, 1-15.

${ }^{41}$ Ediger, M. D.; Harrowell, P.; Yu, L. Crystal Growth Kinetics Exhibit a Fragility-Dependent Decoupling From Viscosity. J. Chem. Phys. 2008, 128, 034709.

42 Magill, J. H. ;Plazek, D. J. Physical Properties of Aromatic Hydrocarbons.II. Solidification

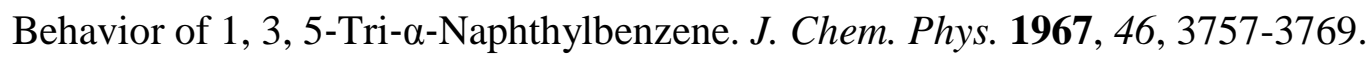

43 Tripathi, P.; Romanini, R.; Tamarit, J. Ll.; Macovez, R. Collective Relaxation Dynamics and Crystallization Kinetics of the Amorphous Biclotymol Antiseptic. Int. J. Pharm. 2015, 495, 420427

44 D’Amore, A. J. M. L.; Kenny, J. M.; Nicolais, L.; Tucci, V. Dynamic-Mechanical and Dielectric Characterization of PEEK Crystallization. Polym. Eng. Sci. 1990, 30, 314-320.

45 Avrami, M. Kinetics of Phase Change. I General Theory. J. Chem. Phys. 1939, 7, 11031112. 
46 Avrami, M. Kinetics of Phase Change. II Transformation-Time Relations for Random Distribution of Nuclei. J. Chem. Phys. 1940, 8, 212-224.

47 Adrjanowicz, K.; et al. Dielectric Relaxation and Crystallization Kinetics of Ibuprofen at Ambient and Elevated Pressure. J. Phys. Chem. B 2010, 114, 6579-6593.

${ }^{48}$ Wunderlich, B. Macromolecular Physics - Crystal Nucleation, Growth, Annealing, Vol. 2, Academic Press: London, 1976.

49 Sterren, V. B.; et al. Preparation of Chloramphenicol/Amino Acid Combinations Exhibiting Enhanced Dissolution Rates and Reduced Drug-Induced Oxidative Stress. AAPS PharmSciTech. 2017, 18, 2910-2918.

${ }^{50}$ Mehta, M.; Ragoonanan, V.; McKenna, G. B.; Suryanarayanan, R. Correlation between Molecular Mobility and Physical Stability in Pharmaceutical Glasses. Mol. Pharm. 2016, 13, $1267-1277$.

51 Mistry, P.; Suryanarayanan, R. Strength of Drug-Polymer Interactions: Implications for Crystallization in Dispersions. Cryst. Growth Des. 2016, 16, 5141-5149.

52 Barták, J; Kostál, P; Podzemná, V; Shánelová, J; Málek, J. Crystal Growth Kinetics and Viscous Behavior in Ge2Sb2Se5 Undercooled Melt. J. Phys. Chem. B 2016, 120, 7998-8006.

53 Sanz, A.; Niss, K. Coupling between Molecular Mobility and Kinetics of Crystal Growth in a Hydrogen-Bonded Liquid. Cryst. Growth Des. 2017, 17, 4628-4636.

${ }^{54}$ Ramos, A. I.; Braga, T. M.; Silva, P.; Fernandes, J. A.; Ribeiro-Claro, P.; Lopes, M.; Paz, F. A. A.; Braga, S. S. Chloramphenicol-cyclodextrin Inclusion Compounds: Co-Dissolution and 
Mechanochemical Preparations and Antibacterial Action. Cryst. Eng. Comm. 2013, 15, 28222834.

55 Mitchell, S. M.; Ullman, J. L.; Teel, A. L.; Watts, R. J. Hydrolysis of Amphenicol and Macrolide Antibiotics: Chloramphenicol, Florfenicol, Spiramycin, and Tylosin. Chemosphere 2015, 134, 504-511.

${ }^{56}$ Franje, C. A.; et al. Differential Heat Stability of Amphenicols Characterized by Structural Degradation, Mass Spectrometry and Antimicrobial Activity. J. Pharm. Biomed. Anal. 2010, 53, 869-877.

57 Steeman, P. A. M.; van Turnhout, J. Fine Structure in the Parameters of Dielectric and Viscoelastic Relaxations. Macromolecules 1994, 27, 5421-5427

58 Wübbenhorst, M.; van Turnhout, J. Analysis of Complex Dielectric Spectra. I. OneDimensional Derivative Techniques and Three-Dimensional Modeling. J. Non-Cryst. Solids 2002, 305, 40-49

59 Ngai, K. L. Relaxation and Diffusion in Complex Systems. Springer Science \& Business Media, 2011.

${ }^{60}$ Ngai, K. L. Relation between Some Secondary Relaxations and the $\alpha$ Relaxations in GlassForming Materials According to the Coupling Model. J. Chem. Phys. 1998, 109, 6982-6994.

61 Koike, T.; Tanaka, R. DielectricProperties above the Glass Transition for a Series of Epoxide Prepolymers. J. Appl. Polym. Sci. 1991, 42, 1333-1340. 
${ }^{62}$ Wojnarowska, Z.; Kołodziejczyk, K.; Paluch, K. J.; Tajber, L., Grzybowska, K.; Ngai, K. L.; Paluch, M. Decoupling of Conductivity Relaxation from Structural Relaxation in ProticIonic Liquids and General Properties. Phys. Chem. Chem. Phys. 2013, 15, 9205-9211.

63 Wojnarowska, Z.; Paluch, M. Recent Progress on Dielectric Properties of Protic Ionic Liquids. J. Phys. Condens. Matter 2015, 27, 073202.

${ }^{64}$ Grohganz, H.; Priemel, P. A.; Löbmann, K.; Nielsen, L. H.; Laitinen, R.; Mullertz, A.; Van den Mooter, G.; Rades, T. Refining Stability and Dissolution Rate of Amorphous Drug Formulations. Expert Opin. Drug Deliv. 2014, 11, 977-989

${ }^{65}$ Lin, Y. N.; Chang, K. M.; Jeng, S. C.; Lin, P. Y.; Hsu, R. Q. Study of Release Speeds and Bacteria Inhibiting Capabilities of Drug Delivery Membranes Fabricated via Electrospinning by Observing Bacteria Growth Curves. J. Mater. Sci. Mater. Med. 2011, 22, 571-577.

${ }^{66}$ Hiraishi, N.; Yiu, C. K. Y.; King, N. M.; Tay, F. R.;Pashley, D. H. Chlorhexidine Release and Water Sorption Characteristics of Chlorhexidine-Incorporated Hydrophobic/Hydrophilic Resins. Dent. Mater. 2004, 24, 1391-1399. 